\title{
Erratum
}

Shoukai Chen, Yongqiwen Fu, Lei Guo*, Shifeng Yang, and Yajing Bie

\section{Statistical Law and Predictive Analysis of Compressive Strength of Cemented Sand and Gravel}

https://doi.org/10.1515/secm-2020-0042

Received Apr 13, 2020; accepted Jul 18, 2020

Erratum to: Shoukai Chen, Yongqiwen Fu, Lei Guo, Shifeng Yang and Yajing Bie, Statistical Law and Predictive Analysis of Compressive Strength of Cemented Sand and Gravel. Science and Engineering of Composite Materials, 27(1), pp. 291-298. Retrieved 13 Apr. 2020, Accepted: 18 Jul., 2020 DOI: https://doi.org/10.1515/secm2020-0030

Authors would like to change Acknowledgment to:

Acknowledgement: This work was supported by the North China University of Water Resources and Electric Power. The authors also would like to acknowledge the support of the National Key R \& D Plan (2018YFC04068 03).

\footnotetext{
*Corresponding Author: Lei Guo: School of Water Conservancy, North China University of Water Resources and Electric Power, Zhengzhou 450045, China; Key Laboratory of Water Environment Simulation and Treatment of Henan Province, Zhengzhou 450002, China; Collaborative Innovation Center of Water Resources Efficient Utilization and Protection Engineering, Zhengzhou 450045, China; Email: glboss@126.com

Shoukai Chen: School of Water Conservancy, North China University of Water Resources and Electric Power, Zhengzhou 450045, China; Key Laboratory of Water Environment Simulation and Treatment of Henan Province, Zhengzhou 450002, China; Collaborative Innovation Center of Water Resources Efficient Utilization and Protection Engineering, Zhengzhou 450045, China Yongqiwen Fu, Shifeng Yang, Yajing Bie: School of Water Conservancy, North China University of Water Resources and Electric Power, Zhengzhou 450045, China
} 